\title{
Illuminating the cells: transient transformation of citrus to study gene functions and organelle activities related to fruit quality
}

\author{
Jinli Gong ${ }^{1,2,3}$, Zhen Tian ${ }^{1,2,3}$, Xiaolu Qu', Qiunan Meng ${ }^{1,2,3}$, Yajie Guan ${ }^{1,2,3}$, Ping Liư ${ }^{4}$, Chuanwu Chen ${ }^{4}$, Xiuxin Deng (1) , \\ Wenwu Guo ${ }^{1}$, Yunjiang Cheng ${ }^{1,2}$ and Pengwei Wang $\mathbb{B}^{1,2,3 凶}$
}

\begin{abstract}
Although multiple microscopic techniques have been applied to horticultural research, few studies of individual organelles in living fruit cells have been reported to date. In this paper, we established an efficient system for the transient transformation of citrus fruits using an Agrobacterium-mediated method. Kumquat (Fortunella crassifolia Swingle) was used; it exhibits higher transformation efficiency than all citrus fruits that have been tested and a prolonged-expression window. Fruits were transformed with fluorescent reporters, and confocal microscopy and livecell imaging were used to study their localization and dynamics. Moreover, various $\mathrm{pH}$ sensors targeting different subcellular compartments were expressed, and the local pH environments in cells from different plant tissues were compared. The results indicated that vacuoles are most likely the main organelles that contribute to the low pH of citrus fruits. In summary, our method is effective for studying various membrane trafficking events, protein localization, and cell physiology in fruit and can provide new insight into fruit biology research.
\end{abstract}

\section{Introduction}

Citrus is one of the most important and highest-yielding fruits in the world, and identifying genes associated with desirable traits is important for the sustainable development of citrus production. However, due to its long juvenile phase and diverse genetic background ${ }^{1}$, obtaining transgenic citrus plants is difficult and time-consuming. Therefore, developing an effective method for the transient transformation of citrus species is important for gene function characterizations and high-throughput screening. The Agrobacterium-mediated infiltration of tobacco leaves is a commonly used method to test gene function in vivo or study protein subcellular localization ${ }^{2}$. However, because of

Correspondence: Pengwei Wang (wangpengwei@mail.hzau.edu.cn)

${ }^{1}$ Key Laboratory of Horticultural Plant Biology (Ministry of Education), College of Horticulture and Forestry Science, Huazhong Agricultural University, 430070 Wuhan, China

${ }^{2}$ National R\&D Centre for Citrus Preservation, Huazhong Agricultural University, 430070 Wuhan, China

Full list of author information is available at the end of the article

These authors contributed equally: Jinli Gong, Zhen Tian their heterogeneous expression, such a system is not ideal for studying genes that are specifically expressed in fruits. Although transient transformation techniques have been tested in fruits, such as strawberry ${ }^{3,4}$, apple $e^{5,6}$, and tomato ${ }^{7}$, none of these techniques proved to be suitable for studying subcellular activities and cell physiology.

Conventionally, cell biological studies in perennial woody plants and fruits have relied on squashing, sectioning, or enzyme-mediated degradation of the cell wall to gain access to the inner compartments. Therefore, conclusions are inevitably obtained from observations of fixed plant tissue, which sometimes does not reflect what truly occurs in planta. For example, the endomembrane compartment is constantly moving and remodeling. It contains the plasma membrane (PM), endoplasmic reticulum (ER), Golgi apparatus, and vacuoles, which play vital roles in protein secretion, storage, and degradation ${ }^{8-10}$. These functional compartments need to maintain a suitable environment; any alterations of their structure, redox potential, $\mathrm{pH}$

\section{(c) The Author(s) 2021}

(c) (i) Open Access This article is licensed under a Creative Commons Attribution 4.0 International License, which permits use, sharing, adaptation, distribution and reproduction cc) in any medium or format, as long as you give appropriate credit to the original author(s) and the source, provide a link to the Creative Commons license, and indicate if changes were made. The images or other third party material in this article are included in the article's Creative Commons license, unless indicated otherwise in a credit line to the material. If material is not included in the article's Creative Commons license and your intended use is not permitted by statutory regulation or exceeds the permitted use, you will need to obtain permission directly from the copyright holder. To view a copy of this license, visit http://creativecommons.org/licenses/by/4.0/. 


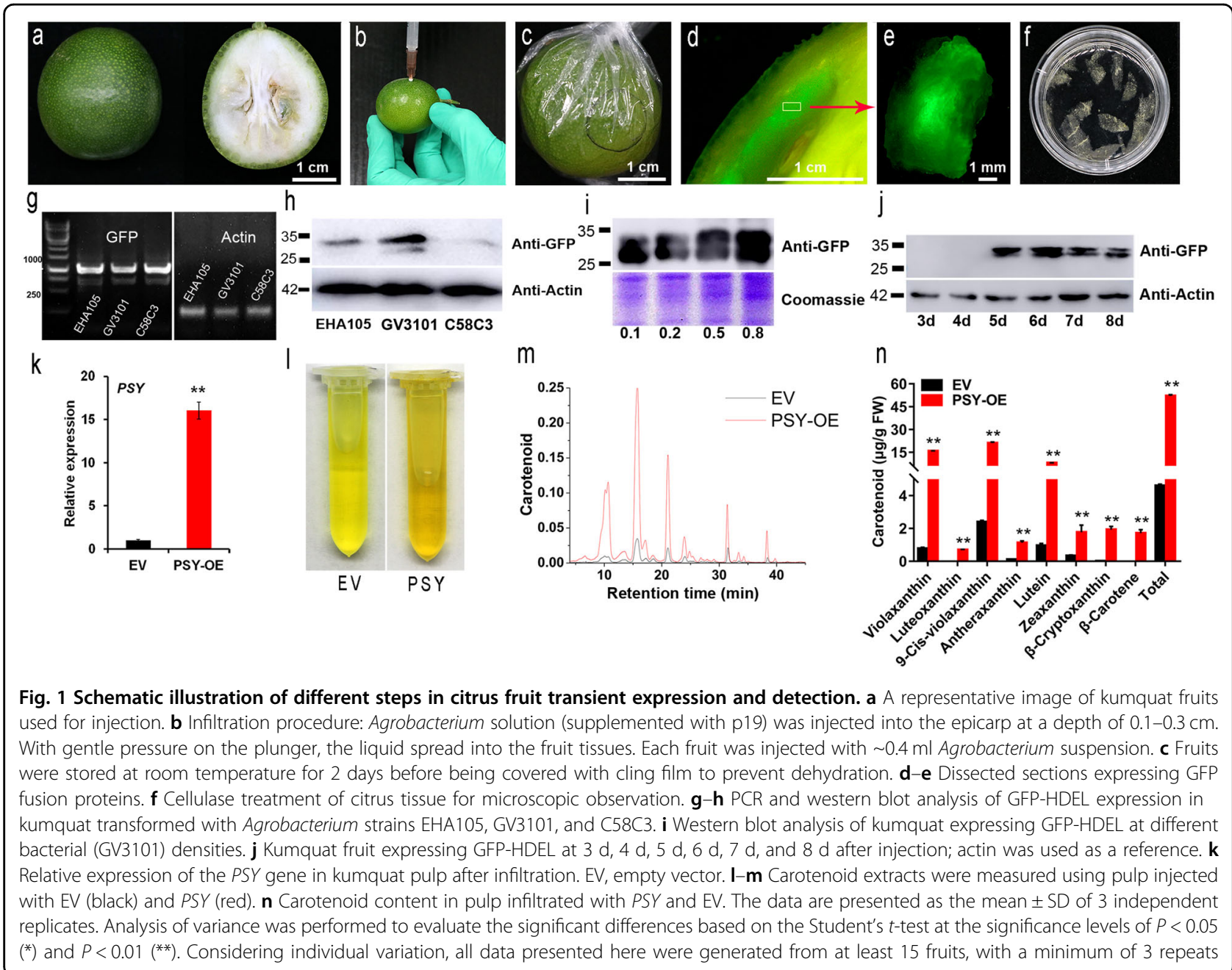

gradient, or lipid composition may lead to severe developmental defects and affect cell viability ${ }^{11,12}$.

However, most of the studies on plant cell biology have been carried out in model systems; little is known about horticultural plants and fruits, which have pleiotropic features that are absent in Arabidopsis and crop species. For example, the local $\mathrm{pH}$ of every organelle is tightly controlled and is critical to its function. Organelle $\mathrm{pH}$ can be measured in vivo using a GFP-derived ratiometric $\mathrm{pH}$ sensor (pHluorin) in tobacco and Arabidopsis ${ }^{13,14}$. For fruits, $\mathrm{pH}$ homeostasis not only affects the cell physiological environment but is also relevant to fruit acidity and quality. Despite its importance, little is known about the $\mathrm{pH}$ environment within most intracellular compartments of fruit cells. For example, fruits of nonacidic Faris lemon had average $\mathrm{pH}$ of 5.8-5.9, whereas those of acidic Faris lemon was 4.0, and those of Frost Lisbon lemon were 3.5-3.6 ${ }^{15}$. Therefore, there must be specific pathways regulating $\mathrm{pH}$ in different fruit varieties. To understand this biological process, it is essential to measure and monitor local $\mathrm{pH}$ at the subcellular level.
Here, kumquat fruits (Fortunella crassifolia Swingle) were selected for Agrobacterium-mediated transient expression. The kumquat is closely related to most major citrus cultivars whose genomic information is widely available. It is also characterized by its high sugar content, thin skin, and relatively few juice cells; these characteristics provide a good environment for infiltration and infection by Agrobacterium. The feasibility of this method was demonstrated by expressing different organelle markers to study protein localization, as well as by expressing pHluorin-derived reporters to measure the $\mathrm{pH}$ of specific compartments of citrus cells. In addition, our results demonstrate that this technique is efficient not only for cell biology studies but also for the functional characterization of genes related to fruit physiology and quality.

\section{Results and discussion}

Agrobacterium-mediated transformation of citrus fruits

The transformation of citrus fruits was carried out in kumquat (Fig. 1a), which is one of the major citrus cultivars in southern China ${ }^{16}$. Kumquat blooms four times a 
year, and fresh fruits are available from November to March of the next year. With good preharvest management and postharvest storage conditions, the period of kumquat availability can be further extended. For the transformation experiment, fruits at the green ripening stage ( 120-180 days after flowering) were used, and they were infiltrated with Agrobacterium tumefaciens carrying an expression vector ( $35 \mathrm{~S}$ promoter-driven). The bacterial solution was gently injected into the epicarp at a depth of $0.1-0.3 \mathrm{~cm}$ (Fig. 1b). The mixture could distribute to an area with a radius of $0.8-1.3 \mathrm{~cm}$ from the injection point, as indicated using a red dye (Supplementary Fig. S1). The infiltrated fruits were stored at room temperature for $\sim 2$ days and wrapped with cling film to prevent dehydration (Fig. 1c). Approximately 5 days after injection, fluorescent signals were clearly visible near the site of injection (Fig. 1d-e). We tested this method using different citrus cultivars, including orange, pomelo, and mandarin (Supplementary Fig. S2) and found that kumquat constantly produced good signals and high transformation efficiency. In addition, fluorescent signals could be detected in different tissues of the kumquat, including the flavedo, albedo, juice sacs, and partition, into which the Agrobacterium solution penetrated (Supplementary Fig. S3).

Moreover, we tested different Agrobacterium tumefaciens strains (e.g., EHA105, GV3101, and C58C3), all of which carried a construct expressing GFP-HDEL (an ER marker). Western blot and PCR results demonstrated that GFP was expressed in all three experiments, and GV3101-mediated transformation produced the highest amount of protein (Fig. 1g-h). The optical density $\left(\mathrm{OD}_{600}\right)$ of Agrobacterium also affects the level of protein expression (Fig. 1i), and it is necessary to optimize the best $\mathrm{OD}_{600}$ for a new construct before the actual study. If potential overexpression artifacts are a major concern, using the lowest $\mathrm{OD}_{600}$ that produces a strong enough signal is recommended. Otherwise, an $\mathrm{OD}_{600}$ of $0.5-0.8$ can be used to provide maximum expression. Moreover, it is also important to keep the injection volume between 0.2 and $0.5 \mathrm{ml}$ for each fruit, as overdoses of infiltration buffer may result in unexpected rotting. In most experiments, protein expression became detectable 4-5 days after infiltration (Fig. 1j). Protein expression could even be detected up to one month after infiltration if the fruits were kept under appropriate conditions (Supplementary Fig. S4). This prolonged-expression pattern was identified in most constructs in our study and could potentially provide a wide time window for certain downstream analyses.

\section{Using the transient expression method for gene characterization}

Fruits may have unique metabolic pathways that are absent in other plant tissues. Therefore, an efficient assay to test the gene function of a particular metabolic pathway could be useful for high-throughput assays. Here, phytoene synthase (PSY), which regulates the most critical step in carotenoid synthesis ${ }^{17,18}$, was selected as an example. The results indicated that the transient overexpression of $P S Y$ resulted in significantly higher expression of $P S Y$ at the mRNA level compared to the control fruits (Fig. 1k), concomitant with carotenoid accumulation (Fig. 1l-n). In addition, with a protein tag (e.g., GFP, HA, Myc), immunoprecipitation (Co-IP) or chromatin immunoprecipitation (ChIP-seq) could be performed to screen possible interacting patterners of citrus protein in its native environment. This is one of the advantages of our system over tobacco expression.

\section{Live cell imaging of citrus fruit cells}

The use of GFP and its derivatives is a landmark in cell biology; it can be fused with genes of interest to study their subcellular localization, dynamics, and protein-protein interactions ${ }^{8}$. To study subcellular activities and protein localization in living citrus fruits, infiltrated fruits were prescreened using a stereomicroscope to identify the successfully transformed area. Tissues with strong fluorescence signals were then hand-sliced into small pieces and transferred to a slide for observation. Alternatively, samples could be incubated in a buffer containing cellulose and macerozyme for $1-2 \mathrm{~h}$ to partially digest the cell wall (Fig. 1f). The main purpose of this additional step was to release the cells from the tissue to facilitate observation. Prolonged digestion can result in the production of protoplasts, but it is normally unnecessary; ideally, it is good to keep any disruptions of this process to a minimum. In addition, it is crucial to minimize mechanical damage and external pressure during the sectioning and imaging of fruit tissues.

Protein localization is one of the key factors involved in understanding protein function in vivo. Here, we infiltrated various organelle markers tagged with GFP/RFP, such as ST-GFP for the Golgi apparatus (Fig. 2a), RFPHDEL for the ER network (Fig. 2b), GFP-Lifeact for the actin cytoskeletons (Fig. 2c), and PT-RFP for the plastids (Fig. 2d). Confocal microscopy suggested that their localization in citrus fruits was consistent with the results from $N$. benthamiana leaf epidermal cells (Supplementary Fig. S5). Next, we infiltrated different markers at the same time to study protein colocalization. For example, H2BGFP and mCherry-HY5 (elongated hypocotyl5, a lightresponsive transcription factor) were co-infiltrated and shown to be colocalized in the nucleus (Fig. 2e). Most organelles and membrane structures can physically interact with each other to form a nexus in eukaryotic cells $^{19,20}$; one of the best examples of this is the ER-Golgi interface $^{21}$. In our study, we coexpressed GFP-HDEL with ST-RFP to label the ER network and Golgi apparatus, and 

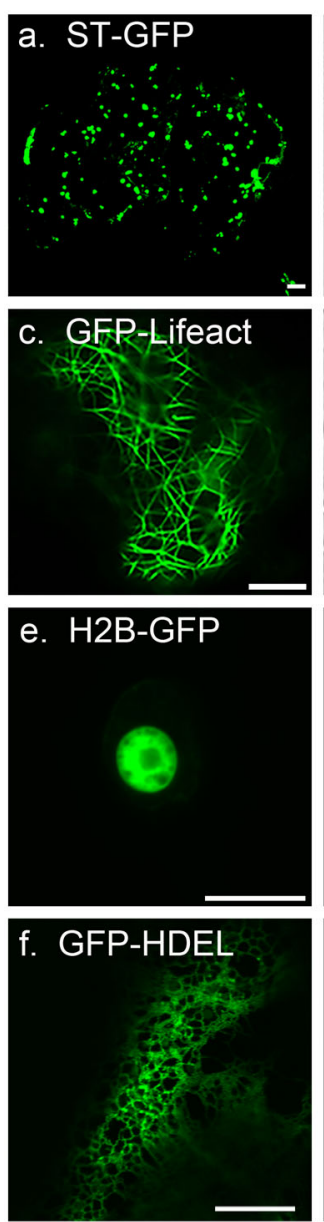
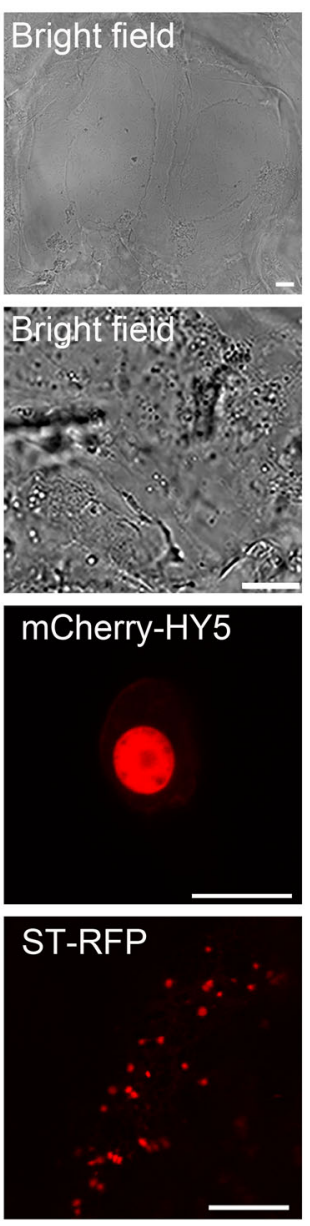
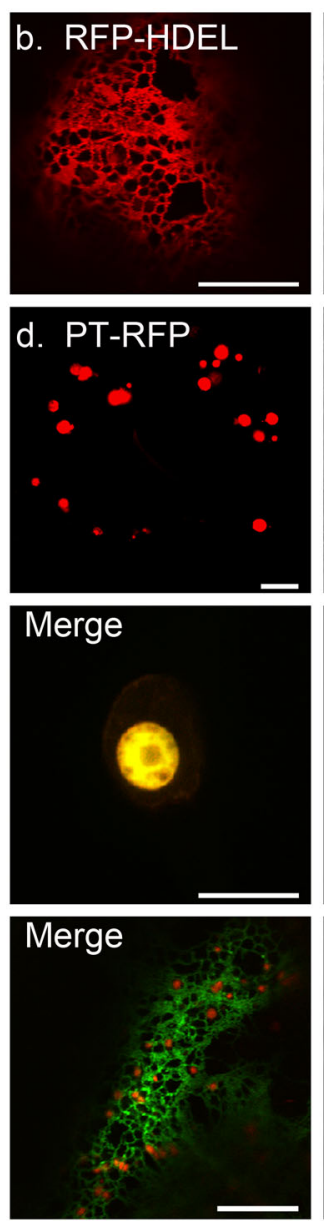
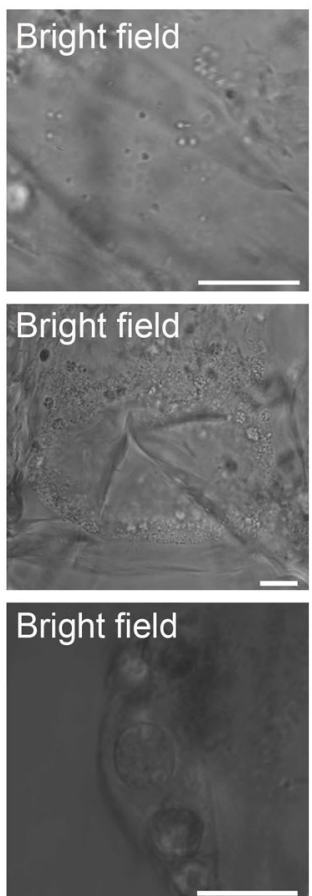

Bright field

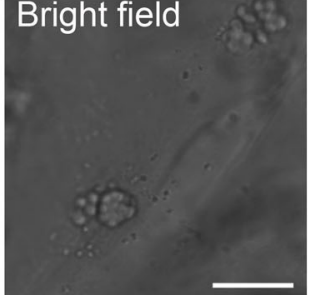

Fig. 2 Transient expression of fluorescent fusion proteins in citrus fruit cells. All constructs were infiltrated at $O D_{600}=0.8$, and images were taken 5 days after infiltration. a-d Representative images of fluorescent protein fusions localized to the Golgi apparatus (ST-GFP), ER (RFP-HDEL), actin cytoskeleton (GFP-Lifeact), and plastids (PT-RFP). e Transient expression of mCherry-HY5 (a transcription factor) localized to the nucleus that was colabeled with H2B-GFP (a nuclear marker). f Coexpression of GFP-HDEL and ST-RFP identified the close association between the ER network and Golgi bodies in kumquat fruit cells. Scale bar, $10 \mu \mathrm{m}$

the results indicated a close association between these structures (Fig. 2f). This finding is consistent with other studies in higher plants ${ }^{22}$.

It is worth pointing out that the structures of the ER and actin cytoskeleton are normally sensitive to mechanical disruption. Their structures remained intact in our experiment, indicating that the manipulation throughout the process caused little disruption to the cells. The Golgi apparatus is the compartment that regulates protein sorting and secretion ${ }^{23}$. In our study, ST-GFP-labeled Golgi bodies exhibited rapid movement, as indicated by our time series images and kymograph (Fig. 3). This result further indicated that live-cell imaging and advanced light microscopy techniques (e.g., BiFC, FRAP, FRET, and ratio imaging) could potentially be applied to study subcellar activities related to fruit physiology.
pHluorin-derived fluorescence ratio imaging demonstrates the local $\mathrm{pH}$ environment at the organelle level

The total acid level is an important measure of fruit quality. As the fruit matures, the acidity of the juice increases, and the $\mathrm{pH}$ decreases. Recent developments in $\mathrm{pH}$-sensitive fluorescent sensors have provided tools to measure the local $\mathrm{pH}$ of different subcellular compartments in vivo ${ }^{13}$. For example, pHluorin is a GFP derivative that can be activated differentially at $405 \mathrm{~nm}$ and $488 \mathrm{~nm}$ lasers depending on the surrounding proton concentration. A higher 405/488 ratio is expected under basic conditions, while a lower 405/488 ratio indicates a more acidic environment. In this study, we used this method to measure the local $\mathrm{pH}$ environment of the apoplast (PMApo), cytoplasm (Cyto-pH), ER (pH-HDEL) network, and vacuole (BCECF-AM). 

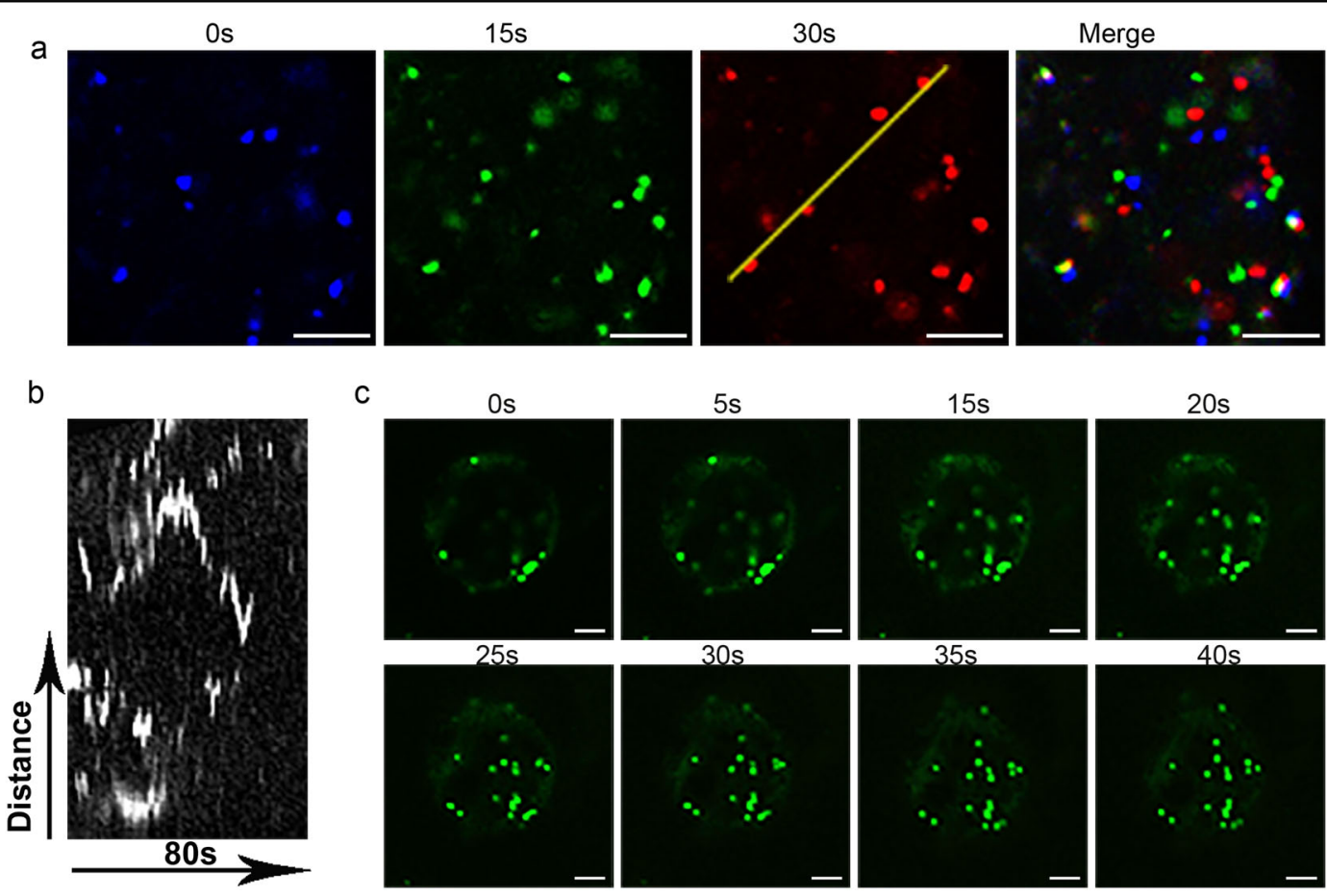

Fig. 3 Live-cell imaging of ST-GFP reveals the dynamic movement of the Golgi bodies in citrus fruit cells. a Time series of ST-GFP-labeled Golgi bodies over $30 \mathrm{~s}$. The images representing the different time points are pseudocolored: blue ( $0 \mathrm{~s})$, green (15 s), and red (30 s). The merged picture with separated signals indicates that the puncta are mobile. b A kymograph was generated along the yellow line, demonstrating the movement of these structures. c Time-lapse images of ST-GFP-labeled Golgi bodies over $40 \mathrm{~s}$ in kumquat cells. Scale bar, $5 \mu \mathrm{m}$

First, His-tagged pHluorin was expressed in E. coli and purified using nickel-agarose beads under nondenaturing conditions. These agarose beads (attached to the pHluorin protein) were used for calibration at a specific $\mathrm{pH}$, and the fluorescence emission ratio at $405 / 488 \mathrm{~nm}$ was measured. A series of measurements were performed under different $\mathrm{pH}$ conditions, and the data were plotted against $\mathrm{pH}$ as a sigmoidal curve ${ }^{24}$ (Boltzmann function, Fig. 4a).

The generated sigmoidal curve was then used to calculate the intracellular $\mathrm{pH}$ in the following experiments. However, pHluorin is not ideal for measuring any $\mathrm{pH}$ below 5 , as its signal tends to be quenched under extremely acidic conditions. To overcome this problem, another fluorescent dye, BCECF-AM, was used in addition to measuring the $\mathrm{pH}$ within the vacuole. Using various $\mathrm{pH}$ buffers $(\mathrm{pH}=3-6)$, the calibration curve was calculated as the fluorescence ratio of BCECF-AM at $488 \mathrm{~nm} / 448 \mathrm{~nm}$ (Fig. 4b).

Before applying these $\mathrm{pH}$ sensors to citrus, we verified their subcellular localization in $N$. benthamiana leaves and measured their $\mathrm{pH}$ environment as described by Martinière et al. ${ }^{13}$. Using our calibration curves, the $\mathrm{pH}$ of the apoplast, cytoplasm, ER network, and vacuole in leaf cells was measured to be $6.4 \pm 0.2,7.4 \pm 0.5,7.3 \pm 0.5$, and $6.0 \pm 0.6$, respectively (Fig. 4). These results are very similar to those in previous studies, indicating that this system works well.

Next, the same studies were performed in citrus. Fruits were transformed as mentioned previously, while citrus calli were transformed using protoplasts by a PEGmediated method. Surprisingly, the measured $\mathrm{pH}$ showed a similar value for the apoplast and small differences in the cytoplasm and ER lumen among leaves, calli, and fruit (Fig. 4). Since significantly different $\mathrm{pH}$ values were found in crude tissue extracts (Fig. 4s), we suspect that this variation may have been caused by the vacuole. To test this hypothesis, the vacuoles were stained with BCECF-AM, and the luminal measurements showed that kumquat fruits exhibited the lowest vacuolar $\mathrm{pH}$ (5.2 \pm 0.4 ), significantly lower than the 5.7 in the callus and the 6.0 in $N$. benthamiana leaves (Fig. 4r).

The unique $\mathrm{pH}$ value of each compartment is essential for protein sorting, as the binding or loading of cargo may rely on the formation of hydrogen bonds with their receptors, and this process is $\mathrm{pH}$ sensitive. Our results show that every organelle in the different plants has a signature $\mathrm{pH}$ (Fig. 5). Most compartments in fruit cells exhibit $\mathrm{pH}$ values similar to those of leaves and calli; however, $\mathrm{pH}$ variation is mainly observed in vacuoles. It is known that the $\mathrm{pH}$ value decreases along the membrane 


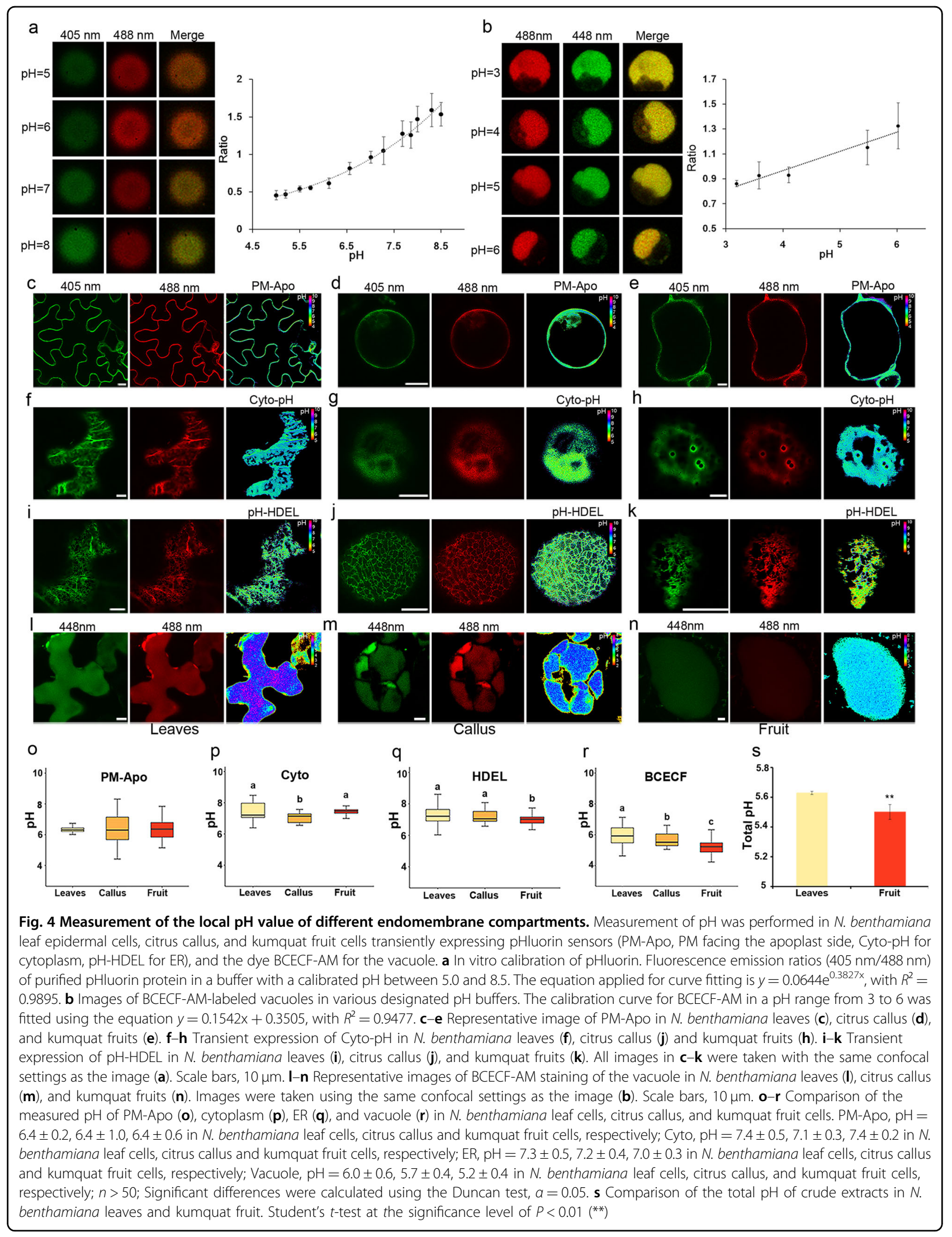



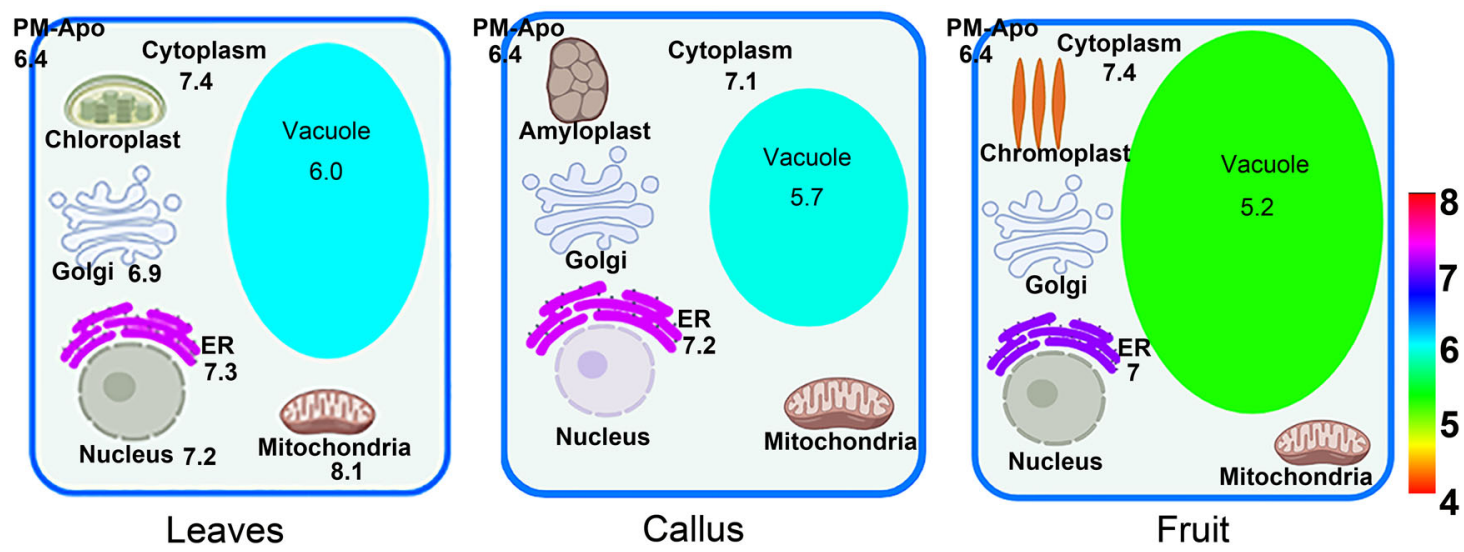

Fig. 5 Comparison of the pH values of intracellular compartments in leaves, calli, and fruit cells. The pH values for the mitochondria, nucleus and Golgi in tobacco leaves were reported previously, and the other $\mathrm{pH}$ measurements were generated from this study

trafficking pathway (ER-Golgi-PVC/MVB-Vacuole); proton pumps that differentially localize to these compartments may work together to achieve such a $\mathrm{pH}$ gradient. However, for fruit varieties with high acid contents, unique vacuolar proton pumps or vacuole biogenesis pathways must be available to generate high proton gradients and create an extremely low $\mathrm{pH}$ environment ${ }^{25}$.

In summary, we studied citrus fruit cells at the subcellular level by using a series of fluorescent organelle markers and $\mathrm{pH}$ reporters. Different sensors (e.g., roGFP for redox potential ${ }^{26}$ ) could also be applied to study the communication between distinct intracellular compartments and specific metabolite (e.g., citrate ${ }^{27}$ ) accumulation at the organelle level in fruit cells. Future studies using this method could potentially be useful to reveal the function and regulatory mechanisms of a particular organelle during fruit development or postharvest.

\section{Materials and methods \\ Plant material}

Kumquat (F. crassifolia Swingle) fruits were collected from Rong'an and Guilin city, Guangxi Province, China. They were sterilized with $2 \%$ sodium hypochlorite solution for $2 \mathrm{~min}$ and rinsed thoroughly in water before Agrobacterium injection.

\section{Plasmid construction and Agrobacterium infiltration}

RNA was extracted from citrus fruits using TRIzol reagent according to the HiPure HP Plant RNA Mini Kit (Magen), and single-stranded cDNA was synthesized by a cDNA Synthesis Kit (Ferment). The PSY and HY5 coding sequences were PCR amplified from the cDNA library of kumquat fruit and cloned using the $\mathrm{pCR}^{\mathrm{Tm}} 8 / \mathrm{GW} / \mathrm{TOPO}$ TA Cloning Kit (Invitrogen). Recombinase reaction was performed using Gateway LR Clonase II Enzyme Mix (Invitrogen) according to the manufacturer's instructions in order to insert the gene into the pMDC43 plasmid for overexpression.
Agrobacterium transformed with a gene construct was grown overnight in liquid LB medium to an O.D. of $0.8-1.0$. Then, they were resuspended to a final O.D. of 0.8 in liquid injection medium containing $0.05 \mathrm{M} \mathrm{MES}$, $2 \mathrm{mM} \mathrm{Na} \mathrm{PO}_{4}, 0.5 \% \quad(\mathrm{~m} / \mathrm{v}) \quad$ D-glucose, and $0.1 \mathrm{mM}$ acetylsyringone.

\section{Analysis of gene and protein expression}

Quantitative analysis of PSY expression levels was performed with a Roche LightCycler 480 system using the $2 \times$ LightCycler 480 SYBR Green master mix (Roche) and a three-step program. The primers were listed in Supplemental Table 1. A total amount of $30 \mu \mathrm{g}$ protein was used for western blot analysis and detected by either GFP or RFP antibodies (Biorbyt) at a dilution of 1:5000 or 1:10,000, respectively. Goat anti-mouse HRP (Biorbyt) was used as the secondary antibody at a dilution ratio of 1:10,000. The fluorescent signals were detected using a chemiluminescent gel imaging system (Amersham Imager 600).

\section{HPLC analysis for carotenoid metabolites}

Carotenoid extraction and quantitation were performed as described previously ${ }^{28}$. Carotenoid metabolites were analyzed by high-performance liquid chromatography (HPLC), which was performed in a Waters liquid chromatography system as described previously ${ }^{29}$. The carotenoids were identified by their characteristic absorption spectra and retention times based on the literature and standards purchased from CaroteNature (Lupsingen, Switzerland). The carotenoids were quantified using calibration curves at $450 \mathrm{~nm}$.

\section{Light microscopy}

Citrus tissues injected with the Agrobacterium suspension were gently placed onto microscope slides and immediately observed with a stereomicroscope (SZX7; Olympus) equipped with a DP70 camera. For confocal 
microscopy, a disposable sharp blade was used to quickly cut the expressed tissue as thinly as possible on microscope slides dipped in water droplets for direct observation. To avoid damage to fruit cells during ultrathin sectioning, alternatively, the samples were digested for $\sim 1-2 \mathrm{~h}$ in a filter-sterilized enzymatic solution containing $1.5 \%$ cellulase R10, $0.4 \%$ macerozyme R1, $0.4 \mathrm{M}$ mannitol, $20 \mathrm{mM} \mathrm{KCl}, 20 \mathrm{mM}$ MES ( $\mathrm{pH}=5.8$ ), and $10 \mathrm{mM} \mathrm{CaCl}_{2}$. The samples were mounted on microscope slides, very carefully sealed with nail polish, and imaged as described previously $^{30}$. All fluorescent protein markers used in this study are listed in Supplemental Table 2.

\section{Protoplast preparation and transfection}

Citrus unshiu callus "Guoqing No. 1" grown for $\sim 20$ days was selected and carefully clamped into a vessel containing the enzymatic solution (same as above) with tweezers. Then, enzymatic hydrolysis was induced by shaking the mixture slightly overnight under dark conditions to obtain enough protoplasts. Protoplast transfection was performed as recommended by Yoo et al. ${ }^{31}$.

\section{Calibration of $\mathrm{pH}$ sensors}

Escherichia coli (BL21) was grown to $\mathrm{OD}_{600}=0.8$ at $37^{\circ} \mathrm{C}$, and $0.1 \mathrm{mM}$ IPTG was added to the bacterial solution to induce the expression of the target protein overnight. Then, the bacteria were collected by centrifugation, washed three times with water, and sonicated in phosphate buffer. The bacterial lysate was centrifuged at $1000 \mathrm{rpm}$ and $4{ }^{\circ} \mathrm{C}$ for $1 \mathrm{~h}$, and the supernatant was collected and filtered through a $0.45 \mu \mathrm{m}$ filter. Next, the target protein was purified using Ni-NTA His Bind Resin following the manufacturer's instructions (Biotech, Wuhan, China). The recombinant pHluorin was diluted in $50 \mathrm{mM}$ MES-KOH buffers with different $\mathrm{pH}$ values. Using a confocal microscope (SP8; Leica), fluorescence signals at emission wavelengths of $505-550 \mathrm{~nm}$ were collected with excitation wavelengths of 405 or $488 \mathrm{~nm}$. The $\mathrm{pH}$ profile was expressed by converting the grayscale ratio image into pseudocolor using ImageJ. All data are shown as the means and standard deviations from at least 10 images for each indicated $\mathrm{pH}$ value. All curve fittings were performed using Origin 9.0 SR1(OriginLab Corp., Northhampton, MA, USA).

\section{Measurement of $\mathrm{pH}$}

To measure the $\mathrm{pH}$ of the specific pHluorin-targeted compartment, samples were excited at 405 or $488 \mathrm{~nm}$ as described above. The parameters of the images taken with CLSM were described by Martinière et al. ${ }^{13}$, and the $\mathrm{pH}$ value was extrapolated from the sigmoidal function obtained from the calibration curve in vitro. The vacuolar $\mathrm{pH}$ was measured with the cell-permeant and $\mathrm{pH}$ sensitive fluorescent dye BCECF-AM according to Tang et al. $^{32}$ with a $505-550 \mathrm{~nm}$ emission bandwidth of the PMT and excitation at 488 or $448 \mathrm{~nm}$. The total pH was calculated by measuring the juice of the leaf and fruit tissues with a pH meter (ST3100, OHAUS).

\section{Acknowledgements}

We thank Anne Osterrieder (Oxford, UK) for proofreading the manuscript. We thank Nadine Paris (Montpellier, France) for providing the various pH sensors used in this study. We thank Haoliang Wan, Meiyan Shi, and Miao Zhang (Huazhong Agricultural University) for their help in this work. This work was supported by the National Key Research and Development Program (2019YFD1000103) and NSFC grants (no. 31772281, 91854102) to P.W.

\section{Author details}

'Key Laboratory of Horticultural Plant Biology (Ministry of Education), College of Horticulture and Forestry Science, Huazhong Agricultural University, 430070 Wuhan, China. ${ }^{2}$ National R\&D Centre for Citrus Preservation, Huazhong Agricultural University, 430070 Wuhan, China. ${ }^{3}$ Interdisciplinary Sciences Research Institute, Huazhong Agricultural University, 430070 Wuhan, China. ${ }^{4}$ Guangxi Academy of Specialty Crops/Guangxi Engineering Research Center of Citrus Breeding and Culture, 541004 Guilin, China

\section{Author contributions}

P.W. and J.G. designed the experiments; J.G. and Z.T. performed the experiments; X.Q., Q.M., Y.G., P.L., and C.C. helped with the experiments; W.G. and X.D. provided citrus materials and provided valuable suggestions throughout the study; P.W. and J.G. wrote the manuscript; and all authors read the final manuscript.

\section{Competing interests}

The authors declare no competing interests.

Supplementary information The online version contains supplementary material available at https://doi.org/10.1038/s41438-021-00611-1.

Received: 11 January 2021 Revised: 14 April 2021 Accepted: 4 May 2021 Published online: 01 August 2021

\section{References}

1. Peña, L. \& Navarro, L. Transgenic citrus. In: Biotechnology in Agriculture And Forestry: Transgenic Trees. (ed. Bajaj Y. P. S.) 39-53 (Springer Verlag, Berlin, 1999).

2. Sparkes, I. A., Runions, J., Kearns, A. \& Hawes, C. Rapid, transient expression of fluorescent fusion proteins in tobacco plants and generation of stably transformed plants. Nat. Protoc. 1, 2019-2025 (2006).

3. Agius, F., Amaya, I., Botella, M. A. \& Valpuesta, V. Functional analysis of homologous and heterologous promoters in strawberry fruits using transient expression. J. Exp. Bot. 56, 37-46 (2005).

4. Hoffmann, T., Kalinowski, G. \& Schwab, W. RNAi-induced silencing of gene expression in strawberry fruit (Fragaria $\mathrm{x}$ ananassa) by agroinfiltration: a rapid assay for gene function analysis. Plant J. 48, 818-826 (2006).

5. An, J.P. et al. EIN3-LIKE1, MYB1, and ETHYLENE RESPONSE FACTOR3 act in a regulatory loop that synergistically modulates ethylene biosynthesis and anthocyanin accumulation. Plant Physiol. 178, 808-823 (2018).

6. Li, T. et al. Apple (Malus domestica) MdERF2 negatively affects ethylene biosynthesis during fruit ripening by suppressing MdACS1 transcription. Plant $\mathrm{J}$. 88, 735-748 (2016)

7. Orzaez, D., Mirabel, S., Wieland, W. H. \& Granell, A. Agroinjection of tomato fruits. A tool for rapid functional analysis of transgenes directly in fruit. Plant Physiol. 140, 3-11 (2006).

8. Hawes, C., Boevink, P., Roberts, A. \& Brandizzi, F. GFP enlightens the study of endomembrane dynamics in plant cells. Plant Biosyst. 135, 3-12 (2001).

9. Robinson, D. G., Brandizzi, F., Hawes, C. \& Nakano, A. Vesicles versus tubes: is endoplasmic reticulum-golgi transport in plants fundamentally different from other eukaryotes? Plant Physiol. 168, 393-406 (2015).

10. Wang, P. W. \& Hussey, P. J. Interactions between plant endomembrane systems and the actin cytoskeleton. Front. Plant Sci. 6, 422 (2015). 
11. Boutte, Y. \& Moreau, P. Modulation of endomembranes morphodynamics in the secretory/retrograde pathways depends on lipid diversity. Curr. Opin. Plant Biol. 22, 22-29 (2014).

12. Chen, J., Stefano, G., Brandizzi, F. \& Zheng, H. Q. Arabidopsis RHD3 mediates the generation of the tubular ER network and is required for Golgi distribution and motility in plant cells. J. Cell Sci. 124, 2241-2252 (2011)

13. Martiniere, A. et al. In vivo intracellular $\mathrm{pH}$ measurements in tobacco and Arabidopsis reveal an unexpected $\mathrm{pH}$ gradient in the endomembrane system. Plant Cell 25, 4028-4043 (2013).

14. Shen, J. B. et al. Organelle $\mathrm{pH}$ in the Arabidopsis endomembrane system. Mol. Plant 6, 1419-1437 (2013).

15. Aprile, $\mathrm{A}$. et al. Expression of the $\mathrm{H}$ plus -ATPase AHA10 proton pump is associated with citric acid accumulation in lemon juice sac cells. Funct. Integr. Genomics 11, 551-563 (2011).

16. Zhu, M. et al. A comprehensive proteomic analysis of elaioplasts from citrus fruits reveals insights into elaioplast biogenesis and function. Hortic. Res. 5, 6 (2018).

17. Fraser, P. D., Enfissi, E. M. A. \& Bramley, P. M. Genetic engineering of carotenoid formation in tomato fruit and the potential application of systems and synthetic biology approaches. Arch. Biochem. Biophys. 483, 196-204 (2009)

18. Paine, J. A. et al. Improving the nutritional value of Golden Rice through increased pro-vitamin A content. Nat. Biotechnol. 23, 482-487 (2005).

19. Stefano, G., Hawes, C. \& Brandizzi, F. ER - the key to the highway. Curr. Opin. Plant Biol. 22, 30-38 (2014).

20. Wang, P. W., Hawes, C. \& Hussey, P. J. Plant endoplasmic reticulum-plasma membrane contact sites. Trends Plant Sci. 22, 289-297 (2017).

21. Hawes, C., Osterrieder, A., Hummel, E. \& Sparkes, I. The plant ER-golgi interface Traffic 9, 1571-1580 (2008).
22. Sparkes, I. A., Ketelaar, T., de Ruijter, N. C. A. \& Hawes, C. Grab a golgi: laser trapping of golgi bodies reveals in vivo interactions with the endoplasmic reticulum. Traffic 10, 567-571 (2009).

23. Boevink, P. et al. Stacks on tracks: the plant Golgi apparatus traffics on an actin/ ER network. Plant J. 15, 441-447 (1998).

24. Schulte, A., Lorenzen, I., Bottcher, M. \& Plieth, C. A novel fluorescent pH probe for expression in plants. Plant Methods 2, 7 (2006).

25. Strazzer, P. et al. Hyperacidification of Citrus fruits by a vacuolar protonpumping P-ATPase complex. Nat. Commun. 10, 744 (2019).

26. Wang, P. et al. KMS1 and KMS2, two plant endoplasmic reticulum proteins involved in the early secretory pathway. Plant J. 66, 613-628 (2011).

27. Hu, Y. et al. A novel "off-on" fluorescent probe based on carbon nitride nanoribbons for the detection of citrate anion and live cell imaging. Sensors 18, 1163 (2018).

28. Cao, H. B. et al. Comprehending crystalline beta-carotene accumulation by comparing engineered cell models and the natural carotenoid-rich system of citrus. J. Exp. Bot. 63, 4403-4417 (2012).

29. Lu, S. W. et al. The citrus transcription factor CsMADS6 modulates carotenoid metabolism by directly regulating carotenogenic genes. Plant Physiol. 176, 2657-2676 (2018)

30. Wang, P. W. \& Hussey, P. J. NETWORKED 3B: a novel protein in the actin cytoskeleton-endoplasmic reticulum interaction. J. Exp. Bot. 68, 1441-1450 (2017).

31. Yoo, S. D., Cho, Y. H. \& Sheen, J. Arabidopsis mesophyll protoplasts: a versatile cell system for transient gene expression analysis. Nat. Protoc. 2, 1565-1572 (2007).

32. Tang, R.-J. et al. Tonoplast calcium sensors CBL2 and CBL3 control plant growth and ion homeostasis through regulating V-ATPase activity in Arabidopsis. Cell Res. 22, 1650-1665 (2012). 